\title{
Energy Detection Based Spectrum Sensing over Enriched Multipath Fading Channels
}

\author{
Alireza Bagheri ${ }^{1}$, Paschalis C. Sofotasios ${ }^{2,3}$, Theodoros A. Tsiftsis ${ }^{4,5}$, Khuong Ho-Van ${ }^{6}$, \\ Michael I. Loupis ${ }^{5}$, Steven Freear ${ }^{7}$, and Mikko Valkama ${ }^{2}$ \\ ${ }^{1}$ New Jersey Institute of Technology, Newark 07102, NJ, USA \\ e-mail: ab745@njit.edu \\ ${ }^{2}$ Tampere University of Technology, 33101 Tampere, Finland \\ e-mail: \{paschalis.sofotasios; mikko.e.valkama\}@tut.fi \\ ${ }^{3}$ Aristotle University of Thessaloniki, 54636 Thessaloniki, Greece \\ ${ }^{4}$ Nazarbayev University, Astana 010000, Kazakhstan \\ e-mail: theodoros.tsiftsis@nu.edu.kz \\ ${ }^{5}$ Technological Educational Institute of Central Greece, 35100 Lamia, Greece \\ e-mail: mloupis@teiste.gr \\ ${ }^{6}$ HoChiMinh City University of Technology, HoChiMinh City, Vietnam \\ e-mail: hvkhuong@hcmut.edu.vn \\ ${ }^{7}$ University of Leeds, LS2 9JT Leeds, UK \\ e-mail: s.freear@leeds.ac.uk
}

\begin{abstract}
Energy detection has been for long constituting the most popular sensing method in RADAR and cognitive radio systems. The present paper investigates the sensing behaviour of an energy detector over Hoyt fading channels, which have been extensively shown to provide rather accurate characterization of enriched multipath fading conditions. To this end, a simple series representation and an exact closed-form expression are firstly derived for the corresponding average probability of detection for the conventional single-channel communication scenario. These expressions are subsequently employed in deriving novel analytic results for the case of both collaborative detection and squarelaw selection diversity reception. The derived expressions have a relatively tractable algebraic representation which renders them convenient to handle both analytically and numerically. As a result, they can be utilized in quantifying the effect of fading in energy detection based spectrum sensing and in the determination of the trade-offs between sensing performance and energy efficiency in cognitive radio communications. Based on this, it is shown that the performance of the energy detector depends highly on the severity of fading as even slight variations of the fading conditions affect the value of the average probability of detection. It is also clearly shown that the detection performance improves substantially as the number of branches or collaborating users increase. This improvement is substantial in both moderate and severe fading conditions and can practically provide full compensation for the latter cases.
\end{abstract}

\section{INTRODUCTION}

Cognitive radio (CR) has been largely proposed as an effective technology of future wireless communications that is capable of mitigating the currently witnessed spectrum scarcity that arises due to the regulated static spectrum allocation and the constantly increasing demands of ubiquitous wireless applications and devices. Based on this, unlicensed secondary users (SUs) may also efficiently utilize available spectrum resources opportunistically along with the licensed primary users (PUs). Spectrum sensing is the process of detecting and monitoring the presence of PUs that occupy licensed frequency bands in order to ensure effective use of these bands by SU's during temporal availability. The primary aim, in this context, is to prevent the creation of interference effects at PUs, which indicates its criticality in CR based communications [1], [2].

Energy detection (ED) is considered a simple and adequate sensing method, compared to other detection schemes, due to its non-coherent structure and low computational and implementation complexities [3]. It is widely known that the operating principle of ED is practically based on a radiometer that measures the energy level of a received signal waveform over an observation time window. The corresponding measure is subsequently compared with a pre-defined energy threshold which then determines whether a PU is present or absent. It is recalled that the detection of unknown signals over a flat band-limited Gaussian noise channel was analyzed by $\mathrm{H}$. Urkowitz in [4], where he derived explicit expressions for the probability of detection, $P_{d}$, and probability of false alarm, $P_{f}$, performance metrics. This problem was also revisited by Kostylev a few decades later [5] who considered quasideterministic signals over multipath fading channels.

Numerous studies have been devoted to the evaluation of the performance of energy detection-based spectrum sensing for different communication and fading scenarios. The authors in [6] derived closed-form expressions for the average probability of detection over Rayleigh, Rice and Nakagami- $m$ fading channels for both single-channel and multi-channel 
scenarios. Similarly, the ED performance in the case of equal gain combining over Nakagami $-m$ multipath fading has been reported by [7] whereas the performance in collaborative spectrum sensing and in relay-based cognitive radio networks was investigated by [8]-[13]. A semi-analytic method for analysing the performance of energy detection of unknown deterministic signals was reported in [15] and is based on the moment-generating function (MGF) method. This method was utilised in the case of maximal-ratio combining (MRC) in the presence of Rayleigh, Rice and Nakagami $-m$ fading as well as for the useful case of correlated Rayleigh and Rician fading channels in [14]-[16]. Finally, the detection of unknown signals in low signal-to-noise-ratio (SNR) over $K$-distributed $(K)$, generalized $K\left(K_{G}\right)$ and the flexible $\eta-\mu, \kappa-\mu$ and mixture gamma fading channels were addressed in [17]-[27].

The Hoyt [28] or Nakagami- $q$ [29] distribution is typically employed to model multipath fading fading conditions owing to strong ionospheric scintillation in satellite links [30] and when mobile satellite channels are modeled as two-state (good/bad) processes. These conditions typically correspond to enriched multipath fading with Hoyt distribution is used to characterize the bad state, while Rician distribution accounts for the good state [31]. The Hoyt fading model is also relatively general as it includes as special cases both one-sided Gaussian fading $(q=0)$ and Rayleigh fading $(q=1)$.

However, in spite of the usefulness of Hoyt fading distribution, no studies relating to the average probability of detection of unknown signals over this type of fading conditions have been reported in the open technical literature. Motivated by this, the present work is devoted to the analytical performance evaluation of ED over Hoyt fading channels by means of the receiver operating characteristics method. Specifically, a novel analytic expression is firstly derived for the average probability of detection of the energy detector in the form of simple infinite series representation. An exact closed-form expression is additionally derived in terms of the generalized Lauricella hypergeometric function and both expressions are subsequently employed in the derivation of respective expressions for the case of square-law selection (SLS) diversity receivers and collaborative based detection.

The derived expressions have a relatively tractable algebraic representation which renders them convenient both analytically and numerically. To this end, they are employed in analyzing the performance of energy detection over various fading scenarios of Hoyt distributed channels. As expected, the ED performance is highly dependent upon the value and variations of the involved fading parameter as well as by the number of diversity branches or collaborative users.

\section{System And Channel Model}

\section{A. Energy Detection Based Sensing Method}

The received signal waveform in narrowband energy detection follows a binary hypothesis that is given by [14, eq. (1)],

$$
r(t)= \begin{cases}n(t) & : H_{0} \\ h s(t)+n(t) & : H_{1}\end{cases}
$$

where $s(t)$ denotes an unknown deterministic signal, $h$ is the complex gain of the channel coefficient an the nd $n(t)$ represents an additive white Gaussian noise (AWGN) process. Furthermore, flat fading The samples of $n(t)$ are assumed to be zero-mean Gaussian random variables with variance $N_{0} W$, with $W$ and $N_{0}$ denoting the single-sided signal bandwidth and a single-sided noise power spectral density, respectively [14]. The hypotheses $H_{0}$ and $H_{1}$ refer to the cases that a signal is absent or present, respectively. The received signal is subject to filtering, squaring and integrating over the time interval $T$ which is given by [6, eq. (2)], namely

$$
y \triangleq \frac{2}{N_{0}} \int_{0}^{T}|r(t)|^{2} d t .
$$

The output of the integrator is a measure of the energy of the received waveform which constitutes a test statistic that determines whether the received energy measure corresponds only to the energy of noise $\left(H_{0}\right)$ or to the energy of both the unknown deterministic signal and noise $\left(H_{1}\right)$. By denoting the observation time bandwidth product as $u=T W$, the test statistic is typically assumed to follow the central chisquare distribution with $2 u$ degrees of freedom under the $H_{0}$ hypothesis and the non central chi-square distribution with $2 u$ degrees of freedom under the $H_{1}$ hypothesis [4]. To this effect, the probability density function (PDF) in the presence of AWGN is expressed as [6, eq. (3)]

$$
p_{Y}(y)= \begin{cases}\frac{1}{2^{u} \Gamma(u)} y^{u-1} e^{-\frac{y}{2}} & : H_{0} \\ \frac{1}{2}\left(\frac{y}{2 \gamma}\right)^{\frac{u-1}{2}} e^{-\frac{y+2 \gamma}{2}} I_{u-1}(\sqrt{2 y \gamma}) & : H_{1}\end{cases}
$$

where $\gamma \triangleq|h|^{2} E_{s} / N_{0}$ is the corresponding instantaneous $\mathrm{SNR}, E_{s}$ is the signal energy while $\Gamma(a)$ and $I_{n}(x)$ denote the Euler's gamma function and the modified Bessel function of the first kind, respectively [32].

As already mentioned, an energy detector is largely characterised by a predefined energy threshold, $\lambda$, which is particularly critical in the decision process and is promptly associated to three measures that overall evaluate the performance of the detector: i) the probability of false alarm, $P_{f}=\operatorname{Pr}\left(y>\lambda \mid H_{0}\right)$; ii) the probability of detection, $P_{d}=\operatorname{Pr}\left(y>\lambda \mid H_{1}\right)$ and iii) the probability of missed detection, $P_{m}=1-\operatorname{Pr}\left(y>\lambda \mid H_{1}\right)=1-P_{d}$. The first two measures are obtained by integrating (3) over the interval between the energy threshold to infinity, $\{\lambda, \infty\}$, yielding [6]

$$
P_{f}=\frac{\Gamma\left(u, \frac{\lambda}{2}\right)}{\Gamma(u)},
$$

and

$$
P_{d}=Q_{u}(\sqrt{2 \gamma}, \sqrt{\lambda}),
$$

where $\Gamma(a, x)$ and $Q_{m}(a, b)$ denote the upper incomplete gamma function and the generalised Marcum $Q$-function, respectively [32], [33]. 


\section{B. The Nakagami-q (Hoyt) Fading Distribution}

Hoyt distribution has been shown to represent effectively the short-term fading effects during wireless transmission. Physically, this fading model considers a signal composed of clusters of multipath waves propagating in a non-homogeneous environment and accounts for the cases that no dominant signal is present and the in-phase and quadrature components of the received signal have different powers, or equivalently, where the in-phase and quadrature components are correlated [29]. The PDF of the Hoyt distribution is given by [34, eq. (2.11)]

$$
p_{\gamma}(\gamma)=\frac{1+q^{2}}{2 q \bar{\gamma}} e^{-\frac{\left(1+q^{2}\right)^{2}}{4 q^{2} \bar{\gamma}} \gamma} I_{0}\left(\frac{1-q^{4}}{4 q^{2} \bar{\gamma}} \gamma\right),
$$

where $q$ is the Nakagami $-q$ fading parameter, which ranges in the interval between zero and unity i.e. $q \in\{0,1\}$.

\section{Spectrum Sensing Performance Analysis}

\section{A. Sensing Performance with No Diversity Reception}

It is recalled that (4) and (5) account for the conventional case of AWGN channels. For communication scenarios over fading channels, it is recalled that the average probability of detection, $\overline{P_{d}}$, is obtained by averaging (5) over the corresponding SNR fading statistics, namely

$$
\overline{P_{d}}=\int_{0}^{\infty} Q_{u}(\sqrt{2 \gamma}, \sqrt{\lambda}) p_{\gamma}(\gamma) d \gamma
$$

Based on this, the average probability of detection in the case of Hoyt fading channels can be obtained by averaging (5) over the statistics of (6). Therefore, substituting (6) into (7) yields

$$
\overline{P_{d}}=\frac{1+q^{2}}{2 q \bar{\gamma}} \int_{0}^{\infty} \frac{Q_{u}(\sqrt{2 \gamma}, \sqrt{\lambda})}{\exp \left(\frac{\left(1+q^{2}\right)^{2}}{4 q^{2} \bar{\gamma}} \gamma\right)} I_{0}\left(\frac{1-q^{4}}{4 q^{2} \bar{\gamma}} \gamma\right) d \gamma .
$$

It is recalled that the Marcum $Q$-function can be expressed in infinite series according to [35, eq. (1.3)], as follows:

$$
Q_{u}(\sqrt{2 \gamma}, \sqrt{\lambda})=1-\sum_{l=0}^{\infty} \frac{\gamma^{l} e^{-\gamma} \gamma\left(l+u, \frac{\lambda}{2}\right)}{\Gamma(l+1) \Gamma(l+u)},
$$

where $\gamma(a, x)=\Gamma(a)-\Gamma(a, x)$ is the lower incomplete gamma function [32]. Thus, by substituting (9) into (8) yields

$$
\begin{aligned}
\overline{P_{d}}=1- & \frac{1+q^{2}}{2 q \bar{\gamma}} \sum_{l=0}^{\infty} \frac{\gamma\left(l+u, \frac{\lambda}{2}\right)}{l ! \Gamma(l+u)} \\
& \times \underbrace{\int_{0}^{\infty} \gamma^{l} e^{-\left(1+\frac{\left(1+q^{2}\right)^{2}}{4 q^{2} \bar{\gamma}}\right) \gamma} I_{0}\left(\frac{1-q^{4}}{4 q^{2} \bar{\gamma}} \gamma\right) d \gamma}_{\mathcal{I}} .
\end{aligned}
$$

To this effect and by expressing the $I_{v}(x)$ function according to [36, eq. (2.216)] and substituting in $\mathcal{I}$, it follows that

$$
\mathcal{I}=\int_{0}^{\infty} \gamma^{l} e^{-\left(1+\frac{\left(1+q^{2}\right)^{2}}{4 q^{2} \bar{\gamma}}\right) \gamma}{ }_{0} F_{1}\left(; 1 ;\left(\frac{1-q^{4}}{8 q^{2} \bar{\gamma}} \gamma\right)^{2}\right) d \gamma
$$

where

$$
{ }_{0} F_{1}(; a ; x) \triangleq \sum_{l=0}^{\infty} \frac{\Gamma(a) x^{l}}{\Gamma(a+l) \Gamma(1+l)}=\sum_{l=0}^{\infty} \frac{1}{(a)_{l}} \frac{x^{l}}{l !}
$$

denotes the confluent hypergeometric function. Notably, the above integral can be expressed in closed-form with the aid of [37, eq. (7.525.1)]. Based on this, $\mathcal{I}$ can be evaluated as

$$
\mathcal{I}=\frac{\Gamma(l+1){ }_{2} F_{1}\left(\frac{l+1}{2}, \frac{l+2}{2} ; 1 ;\left(\frac{1-q^{4}}{4 q^{2} \bar{\gamma}+\left(1+q^{2}\right)^{2}}\right)^{2}\right)}{\left(1+\frac{\left(1+q^{2}\right)^{2}}{4 q^{2} \bar{\gamma}}\right)^{l+1}}
$$

where

$$
{ }_{2} F_{1}(a, b ; c ; x) \triangleq \sum_{l=0}^{\infty} \frac{\Gamma(c) \Gamma(a+l) \Gamma(b+l) x^{l}}{\Gamma(a) \Gamma(b) \Gamma(c+l) \Gamma(1+l)}
$$

denotes the Gauss hypergeometric function [32]. Hence, by substituting (13) into (10) yields the following infinite series

$$
\begin{aligned}
\overline{P_{d}}=1- & \sum_{l=0}^{\infty} \frac{\left(1+q^{2}\right) \gamma\left(l+u, \frac{\lambda}{2}\right)}{2 q \bar{\gamma}\left(1+\frac{\left(1+q^{2}\right)^{2}}{4 q^{2} \bar{\gamma}}\right)^{l+1} \Gamma(l+u)} \\
& \times{ }_{2} F_{1}\left(\frac{l+1}{2}, \frac{l+2}{2} ; 1 ;\left(\frac{1-q^{4}}{4 q^{2} \bar{\gamma}+\left(1+q^{2}\right)^{2}}\right)^{2}\right) .
\end{aligned}
$$

The above series has a convenient algebraic representation and it is fully convergent while it exhibits low truncation error after relatively few terms. As a result, it can be evaluated rather straightforwardly while it can be also used as a basis to derive an exact closed-form expression in terms of the generalized Lauricella function. To this end, by expanding the Gaussian confluent hypergeometric function and the lower incomplete gamma function according to (14) and [37, eq. (8.354.1)], respectively, it readily follows that

$$
\begin{aligned}
\overline{P_{d}}=1- & \sum_{l=0}^{\infty} \sum_{i=0}^{\infty} \sum_{j=0}^{\infty} \frac{2^{1-u+l-j}(-1)^{j} \lambda^{u+j}\left(q^{2} \bar{\gamma} \lambda\right)^{l}}{j !(i !)^{2}\left(4 q^{2} \bar{\gamma}+\left(1+q^{2}\right)^{2}\right)^{1+l+2 i}} \\
& \times \frac{\left(1-q^{2}\right)^{2 i}\left(1+q^{2}\right)^{1+2 i} \Gamma\left(\frac{1+l}{2}+i\right) \Gamma\left(\frac{2+l}{2}+i\right)}{(u+l+j) \Gamma(u+l) \Gamma\left(\frac{1+l}{2}\right) \Gamma\left(\frac{2+l}{2}\right)} .
\end{aligned}
$$

Importantly, with the aid of the Legendre duplication formula $\Gamma(a) \Gamma\left(a+\frac{1}{2}\right) \triangleq \sqrt{\pi} 2^{1-2 a} \Gamma(2 a)$, the gamma functions in (16) can be expressed as follows

$$
\Gamma\left(\frac{1+l}{2}+i\right) \Gamma\left(\frac{2+l}{2}+i\right)=\frac{\sqrt{\pi}}{2^{l+2 i}} \Gamma(1+l+2 i)
$$

and

$$
\Gamma\left(\frac{1+l}{2}\right) \Gamma\left(\frac{2+l}{2}\right)=\frac{\sqrt{\pi}}{2^{l}} \Gamma(1+l) .
$$

Furthermore, the $(u+l+j)$ term in (16) can be also expressed as $u+l+j=\Gamma(1+u+l+j) / \Gamma(u+l+j)$. By 
recalling that the Pochhammer symbol is defined as $(a)_{n} \triangleq$ $\Gamma(a+n) / \Gamma(a)$, eq. (16) can be equivalently re-written as

$$
\begin{aligned}
\overline{P_{d}}=1-c_{1} & \sum_{l=0}^{\infty} \sum_{i=0}^{\infty} \sum_{j=0}^{\infty} \frac{(u)_{l+j}(1)_{l+2 i}}{(1+u)_{l+j}(u)_{l}(1)_{i}} \\
& \times \frac{\left(\frac{2 q^{2} \bar{\gamma} \lambda}{4 q^{2} \bar{\gamma}+\left(1+q^{2}\right)^{2}}\right)^{l}}{l !} \frac{\left(\frac{1-q^{4}}{8 q^{2} \bar{\gamma}+2\left(1+q^{2}\right)^{2}}\right)^{2 i}}{i !} \frac{\left(-\frac{\lambda}{2}\right)^{j}}{j !},
\end{aligned}
$$

where

$$
c_{1}=\frac{2^{1-u} \lambda^{u}\left(1+q^{2}\right)}{u !\left(4 q^{2} \bar{\gamma}+\left(1+q^{2}\right)^{2}\right)} .
$$

Based on this, the average probability of detection over Hoyt fading channels can be expressed in closed-form according to (21), at the top of the next page, where $F_{C: D^{\prime} ; \ldots ; D^{(n)}}^{A: B^{\prime} ; \ldots ; B^{(n)}}$ denotes the Lauricella hypergeometric function of $n$ variables.

\section{B. Sensing Performance with Collaborative Scenario}

It is recalled that the performance of energy detection is substantially enhanced when it is performed in the context of collaborative detection. Based on this, the corresponding average probability of detection and probability of false alarm for the case of $n$ collaborative users are defined as $Q_{d} \triangleq$ $1-\left(1-P_{d}\right)^{n}$ and $Q_{f} \triangleq 1-\left(1-P_{f}\right)^{n}$ [9]. To this effect and after basic algebraic manipulations, the corresponding average probability of detection for $n$ number of collaborative users is given by (22), at the top of the next page.

\section{Sensing Performance with Diversity Reception}

Diversity reception has been also largely shown to provide significant improvement of the performance of energy detection. Among others, SLS reception is distinct for its simplicity and adequate performance. Thus, in the case of energy detection over Hoyt fading channels, the respective average probability of detection is obtained by averaging $P_{\mathrm{d}, \mathrm{SLS}}$ in [6, eq. (15)] over $L$ independent Hoyt distributed branches, which yields

$$
{\overline{P_{d}}}^{\mathrm{SLS}}=1-\prod_{i=1}^{L}\left\{1-\overline{P_{d}}\left(\gamma_{i}\right)\right\} .
$$

To this effect, by substituting (21) in (23), one obtains the closed-form representation in (24), at the top of the next page.

As always, the corresponding $P_{f}$ is independent of the fading statistics and is given by [6, eq. (14)]

$$
{\overline{P_{f}}}^{\mathrm{SLS}}=P_{f}{ }^{\mathrm{SLS}}=1-\left[1-\frac{\Gamma\left(u, \frac{\lambda}{2}\right)}{\Gamma(u)}\right]^{L}
$$

which is expressed by a simple closed-form representation.

To the best of the authors knowledge, the offered results have not been previously reported in the open technical literature.

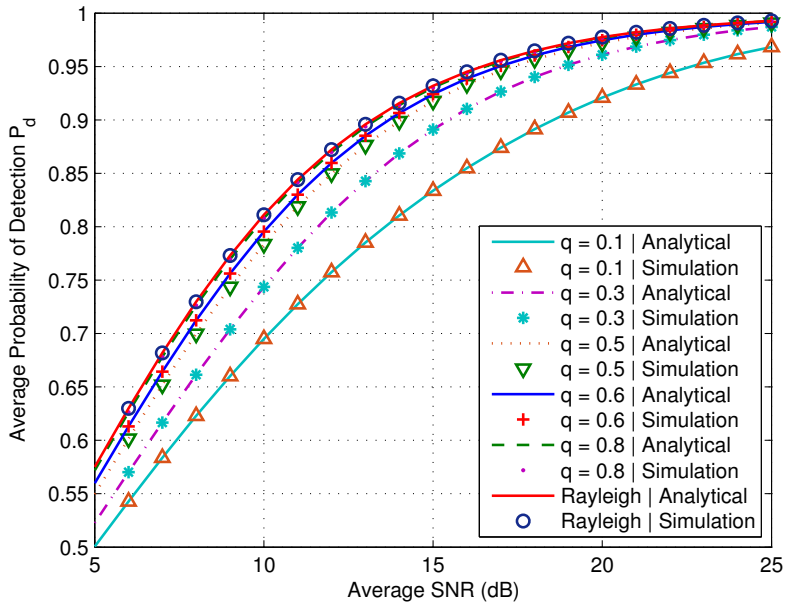

Fig. 1. $\bar{P}_{d}$ vs $\bar{\gamma}$ for i.i.d Hoyt fading with $P_{f}=0.1, u=5$, and different values of $q$.

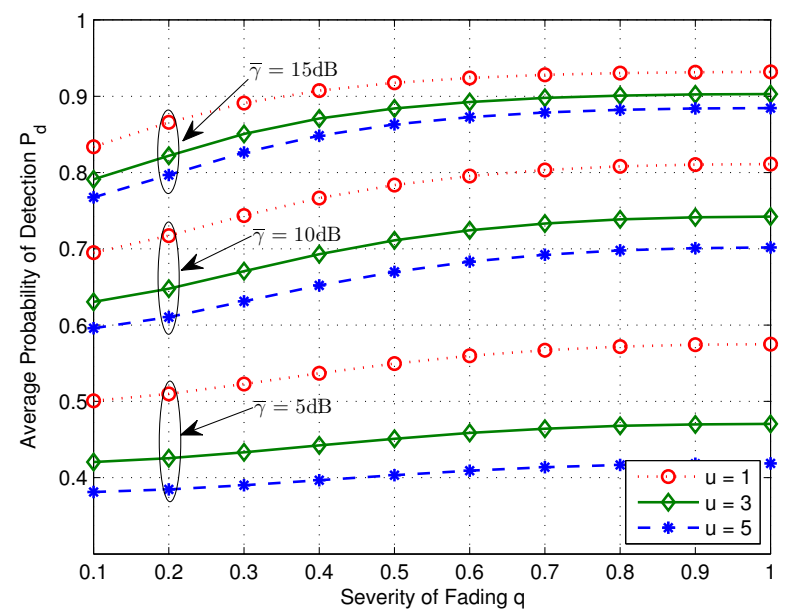

Fig. 2. $\bar{P}_{d}$ vs $q$ for i.i.d Hoyt fading with $P_{f}=0.1$ and different values of $u$ and $\bar{\gamma}$.

\section{NumericAl Results}

Having derived novel analytic results for the average probability of detection, this section is devoted to the analysis of the behaviour of energy detection over enriched fading conditions modeled by Hoyt fading distribution. The corresponding performance is evaluated for different communication scenarios of interest through both $\bar{P}_{d}$ versus $\bar{\gamma}$ curves and complementary receiver operating characteristics (ROC) curves $\left(P_{m}\right.$ versus $\left.P_{f}\right)$. In addition, the effect of the fading parameter $q$ on the value of $P_{d}$ is numerically quantified.

Figs. 1-2 demonstrate the effect of the fading parameter on the sensing performance of energy detection over Hoyt fading channels. Specifically, Fig. 1 presents the behaviour of $\bar{P}_{d}$ vs $\bar{\gamma}$ curves for different values of fading severity $q$ with $P_{f}=0.1$ and $u=5$. One can observe the significant deviation of $\bar{P}_{d}$ for severe fading $(0 \leq q \leq 0.5)$ conditions and slight deviation 


$$
\overline{P_{d}}=1-c_{1} F_{1: 1 ; 1 ;}^{2: ; ;}\left[\begin{array}{l|l}
(u: 1,0,1),(1: 1,2,0): ; ; \\
(1+u: 1,0,1):(u: 1) ;(1: 1) ; & \frac{2 q^{2} \bar{\gamma} \lambda}{4 q^{2} \bar{\gamma}+\left(1+q^{2}\right)^{2}},\left(\frac{1-q^{4}}{8 q^{2} \bar{\gamma}+2\left(1+q^{2}\right)^{2}}\right)^{2},-\frac{\lambda}{2}
\end{array}\right]
$$

$$
\overline{Q_{d}}=1-\left[c_{1} F_{1: 1 ; 1 ;}^{2: ; ;}\left[\begin{array}{l}
(u: 1,0,1),(1: 1,2,0): ; ; \\
(1+u: 1,0,1):(u: 1) ;(1: 1) ;
\end{array} \mid \frac{2 q^{2} \bar{\gamma} \lambda}{4 q^{2} \bar{\gamma}+\left(1+q^{2}\right)^{2}},\left(\frac{1-q^{4}}{8 q^{2} \bar{\gamma}+2\left(1+q^{2}\right)^{2}}\right)^{2},-\frac{\lambda}{2}\right]\right]^{n}
$$

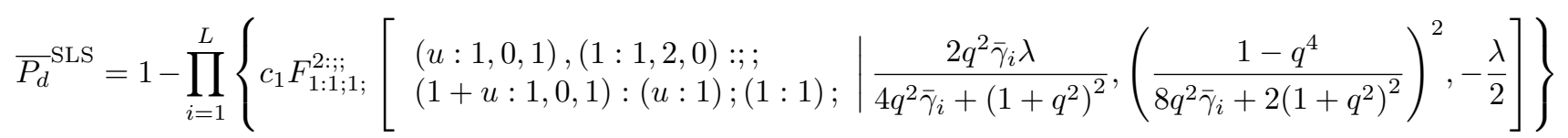

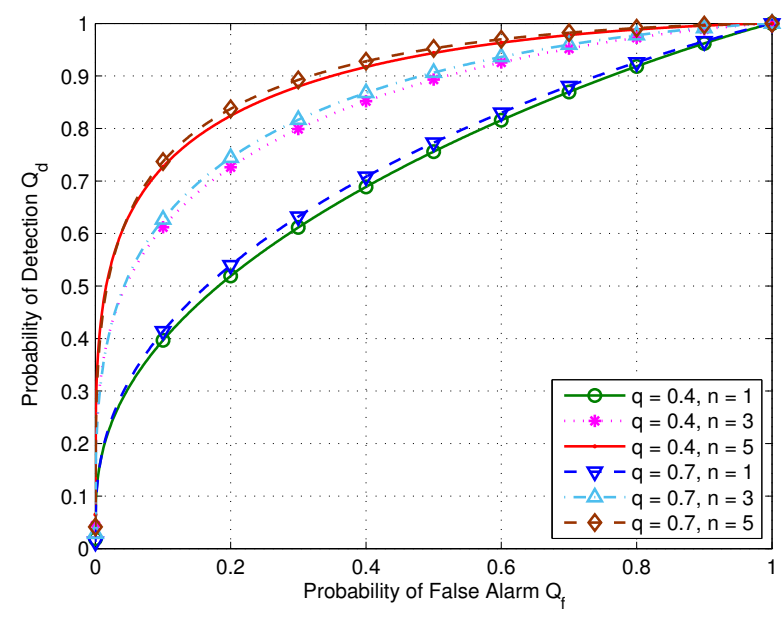

Fig. 3. ROC curves for Hoyt fading with different $q$ values, $u=5$, $\bar{\gamma}=5 \mathrm{~dB}$ and $n$ collaborative users.

for non-severe fading $(0.5<q \leq 1)$ cases. For example, for the case of $\bar{\gamma}=12 \mathrm{~dB}$, the $\bar{P}_{d}$ for $q=0.5$ is nearly $12 \%$ higher than for $q=0.1$. In the same context, when $\bar{\gamma}=10 \mathrm{~dB}$, the $\bar{P}_{d}$ is $2 \%$ higher for $q=0.8$ than for $q=0.6$, whereas the special of $q=1$, which coincides with that for Rayleigh fading channels is also demonstrated. Moreover, Fig. 2 depicts the behavior of $\bar{P}_{d}$ vs $q$ for $P_{f}=0.1$ and different values of $u$ and $\bar{\gamma}$. Yet, the energy detector exhibits better performance for higher $\bar{\gamma}$ and lower $u$. For instance, for the case of $q=0.4$ and $u=3$, the $\bar{P}_{d}$ for $\bar{\gamma}=10 \mathrm{~dB}$ is nearly $57 \%$ higher than for $\bar{\gamma}=5 \mathrm{~dB}$. Likewise, when $q=0.7$ and $\bar{\gamma}=5 \mathrm{~dB}$, the $\bar{P}_{d}$ is $37 \%$ lower for $u=5$ than for $u=1$.

Fig. 3 illustrates the performance of the detector in the case of collaborating sensing considering $q=0.4$ and $q=0.7$ with $u=5, \bar{\gamma}=5 \mathrm{~dB}$ and $n=1, n=3, n=5$. It is evident that the effects of fading are crucial on the performance of the energy detector which is substantially improved as the number of collaborating users increases. For example, for the case of $q=0.4$ and $Q_{f}=0.1$, the $Q_{d}$ for $n=5$ and $n=3$ is

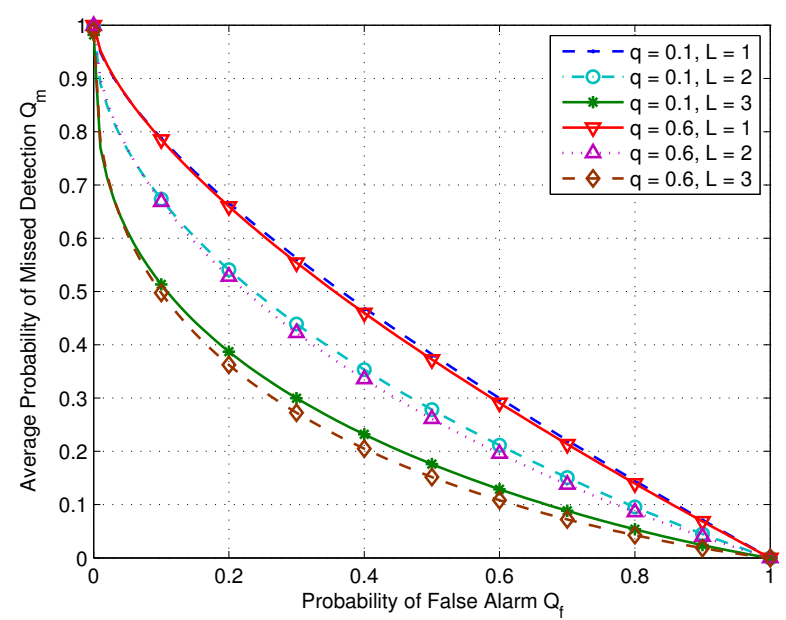

Fig. 4. Complementary ROC curves for SLS diversity reception over Hoyt fading with $u=1, \overline{\gamma_{1}}=-3 \mathrm{~dB}, \overline{\gamma_{2}}=-0 \mathrm{~dB}, \overline{\gamma_{3}}=3 \mathrm{~dB}$ and $L$ branches.

nearly $83 \%$ and $54 \%$ higher compared to the conventional non-collaborative scenario $(n=1)$, respectively. In the same context, Fig. 4 shows complementary ROC curves for the SLS diversity scheme where we assume $u=1, \overline{\gamma_{1}}=-3 \mathrm{~dB}, \overline{\gamma_{2}}=$ $-0 \mathrm{~dB}, \overline{\gamma_{3}}=3 \mathrm{~dB}$ and $L$ number of branches. It is clearly seen that the value of $P_{m}$ is affected significantly as the number of diversity branches increase even in severe fading conditions.

\section{CONCLUSION}

The present work analysed the performance of energy detection based sensing method over enriched fading channels modeled by Hoyt fading distribution. Novel closed-form and analytical expressions were derived for the average probability of detection and was extensively shown that the overall performance of the detector is largely affected by the severity of the enriched multipath fading conditions. This is evident by the fact that even slight variations of the Hoyt fading parameter results to substantial variation of the corresponding 
probability of detection. The offered results were also extended in collaborating spectrum sensing scenarios as well as in SLS diversity which were shown to increase significantly the performance of the detector. The proposed analytic results have a relatively simple algebraic representation and therefore, they can be used in quantifying the effect of fading in energy detection spectrum sensing and can contribute towards improved and/or more energy efficient cognitive radio-based wireless communications.

\section{ACKNOWLEDGEMENTS}

This research is funded by the Research Program DGRES (MIS 380360) within the Research Activity ARCHIMEDES III, funded by the NSRF 20072013, Greece, by the Vietnam National Foundation for Science and Technology Development (NAFOSTED) under grant number 102.04-2014.42, by the Finnish Funding Agency for Technology and Innovation (Tekes), under the project entitled Energy-Efficient Wireless Networks and Connectivity of Devices - Systems (EWINE-S) and by the UK Engineering and Physical Sciences Research Council (EPSRC).

\section{REFERENCES}

[1] S. Haykin, "Spectrum sensing for cognitive radio", Proc. IEEE, vol. 97, no. 5, pp. 849-877, May 2009.

[2] A. Bagheri, A. Shahini, and A. Shahzadi, "Analytical and learning-based spectrum sensing over channels with both fading and shadowing," in Proc. IEEE Int. Conf. on Connected Vehicles and Expo (ICCVE), pp. 699-706, Dec. 2013.

[3] T. Yücek and H. Arslan, "A survey of spectrum sensing algorithms for cognitive radio applications", Commun. Surveys Tuts., vol. 11, no. 1, pp. 116-130, 2009.

[4] H. Urkowitz, "Energy detection of unknown deterministic signals", Proc. IEEE, vol. 55, no. 4, pp. 523-531, 1967.

[5] V. I. Kostylev, "Energy detection of signal with random amplitude", in Proc. IEEE ICC '02, pp. 1606-1610, May 2002.

[6] F. F. Digham, M. S. Alouini, and M. K. Simon, "On the energy detection of unknown signals over fading channels", IEEE Trans. Commun. vol. 55, no. 1, pp. 21-24, Jan. 2007.

[7] S. P. Herath and N. Rajatheva, "Analysis of equal gain combining in energy detection for cognitive radio over Nakagami channels", in Proc. IEEE Globecom '08, pp. 2972-2976, Dec. 2008.

[8] A. Ghasemi and E.S. Sousa, "Collaborative spectrum sensing for opportunistic access in fading environments", in Proc. DySpan '05, pp. 131-136, Nov. 2005

[9] A. Ghasemi and E.S. Sousa, "Impact of User Collaboration on the Performance of Sensing-Based Opportunistic Spectrum Access", in Proc. IEEE Vehicular Tech. Conf. (VTC-fall '06), pp. 1-6, Sep. 2006.

[10] A. Ghasemi and E.S. Sousa, "Asymptotic Performance of collaborative spectrum sensing under correlated Log-normal shadowing", IEEE Comm. Lett., vol. 11, no. 1, pp. 34-36, Jan. 2007.

[11] S. Atapattu, C. Tellambura, and H. Jiang, "Relay based cooperative spectrum sensing in cognitive radio networks", in Proc. IEEE Global Telecomm. Conf. (Globecom '09), pp. 4310-4314, Nov. 2009.

[12] S. Atapattu, C. Tellambura, and H. Jiang, "Energy detection based cooperative spectrum sensing in cognitive radio networks", IEEE Trans. Wireless Commun., vol. 10, no. 4, pp. 1232-1241, Apr. 2011.

[13] A. Shahini, A. Bagheri, and A. Shahzadi, "A unified approach to performance analysis of energy detection with diversity receivers over Nakagami-m fading channels", in Proc. IEEE Int. Conf. on Connected Vehicles and Expo (ICCVE), pp. 707-712, Dec. 2013.

[14] K. T. Hemachandra and N. C. Beaulieu, "Novel analysis for performance evaluation of energy detection of unknown deterministic signals using dual diversity", in Proc. IEEE Vehicular Tech. Conf. (VTC-fall '11), pp. 1-5, Sep. 2011.
[15] S. P. Herath, N. Rajatheva, and C. Tellambura, "Energy detection of unknown signals in fading and diversity reception", IEEE Trans. Commun., vol. 59, no. 9, pp. 2443-2453, Sep. 2011.

[16] A. Bagheri and A. Shahzadi, "Another look at performance analysis of energy detector with multichannel reception in Nakagami-m fading channels", Wireless Pers. Commun., vol. 79, no. 1, pp. 527-544, 2014.

[17] K. Ruttik, K. Koufos, and R. Jantti, "Detection of unknown signals in a fading environment", IEEE Comm. Lett., vol. 13, no. 7, pp. 498-500, July 2009.

[18] S. Atapattu, C. Tellambura, and H. Jiang, "Performance of an energy detector over channels with both multipath fading and shadowing", IEEE Trans. Wireless Commun., vol. 9, no. 12, pp. 3662-3670, Dec. 2010.

[19] S. Atapattu, C. Tellambura, and H. Jiang, "Energy Detection of Primary signals over $\eta-\mu$ fading channels", in Proc. $4^{\text {th }}$ Ind. Inf. Systems (ICIIS '09), pp. 1-5, Dec. 2009.

[20] P. C. Sofotasios, E. Rebeiz, L. Zhang, T. A. Tsiftsis, D. Cabric, and S. Freear, "Energy Detection-Based Spectrum Sensing over $\kappa-\mu$ and $\kappa-\mu$ Extreme Fading Channels", IEEE Trans. Veh. Techn., vol. 63, no 3, pp. 1031-1040, Mar. 2013.

[21] P. C. Sofotasios, M. K. Fikadu, K. Ho-Van, and M. Valkama, "Energy detection sensing of unknown signals over Weibull fading channels," IEEE ATC '13, pp. 414-419, Oct. 2013.

[22] K. Ho-Van, P. C. Sofotasios, G. C. Alexandropoulos, and S. Freear, "Bit error rate of underlay decode-and-forward cognitive networks with best relay selection," IEEE/KICS J. Commun. Networks, vol. 17, no. 2, pp. 162-171, Apr. 2015.

[23] P. C. Sofotasios, S. Muhaidat, G. K. Karagiannidis, and B. S. Sharif, "Solutions to integrals involving the Marcum $Q$-function and applications," IEEE Signal Process. Lett., vol. 22, no. 10, pp. 1752-1756, Oct. 2015.

[24] A. Bagheri, P. C. Sofotasios, T. A. Tsiftsis, A. Shahzadi, S. Freear, and M. Valkama, "Area under ROC curve of energy detection over generalized fading channels," in IEEE PIMRC '15, Hong Kong, Aug/Sep. 2015.

[25] O. Alhussein, A. Al Hammadi, P. C. Sofotasios, S. Muhaidat, J. Liang, M. Al-Qutayri, and G. K. Karagiannidis, "Performance analysis of energy detection over mixture gamma based fading channels with diversity reception," in IEEE WiMob '15, Abu Dhabi, UAE, Oct. 2015.

[26] A. Al Hammadi, O. Alhussein, P. C. Sofotasios, S. Muhaidat, M. AlQutayri, S. Al-Araji, G. K. Karagiannidis, and J. Liang, "Unified analysis of cooperative spectrum sensing over composite and generalized fading channels," IEEE Trans. on Veh. Technol., To appear.

[27] P. C. Sofotasios, L. Mohjazi, S. Muhaidat, M. Al-Qutayri, and G. K. Karagiannidis, "Energy detection of unknown signals over cascaded fading channels," IEEE Antennas Wireless Propag. Lett., To appear.

[28] R. S. Hoyt, "Probability functions for the modulus and angle of the normal complex variate", Bell Syst. Tech. J., vol. 26, no. 2, pp. 318-359, April 1947.

[29] M. Nakagami, "The m-distribution: A general formula of intensity distribution of rapid fading", in Statistical Methods in Radio Wave Propagation, W. C. Hoffman, Ed. Oxford, U.K.: Pergamon, pp. 3-36, 1960.

[30] B. Chytil, "The distribution of amplitude scintillation and the conversion of scintillation indices", J. Atmos. Terr. Phys., vol. 29, no. 9, pp. 1175-1177, Sept. 1967.

[31] A. Chandra, C. Bose, and M. K. Bose, "Performance of non-coherent MFSK with selection and switched diversity over Hoyt fading channel", Wireless Pers. Commun., vol. 68, no. 2, pp. 379-399, 2003.

[32] M. Abramowitz and I. A. Stegun, "Handbook of mathematical functions with formulas, graphs, and mathematical tables.", New York: Dover, 1974.

[33] J. I. Marcum, "A statistical theory of target detection by pulsed radar: Mathematical appendix", RAND Corp., Santa Monica, Research memorandum, CA, 1948.

[34] M. K. Simon and M. -S. Alouni, "Digital Communication over Fading Channels", 2nd Edition, New York: Wiley, 2005.

[35] N. M. Temme, "Asymptotic and numerical aspects of the noncentral chi-square distribution", Computers \& Mathematics with Applications, vol. 25, no. 5, pp. 55-63, 1993.

[36] M. A. Chaudhry and S. M. Zubair, On a class of incomplete Gamma function with applications. Boca Raton-London-Ney York-Washington, D.C.: Chapman \& Hall/CRC, 2002.

[37] I. S. Gradshteyn and I. M. Ryzhik, "Table of Integrals, Series, and Products", $7^{\text {th }}$ ed. New York: Academic, 2007. 